\title{
Intraarticular Injection of Different Doses of Mesenchymal Stem Cell Derived Exosomes Reduces ATF-3 Expression in the Dorsal Root Ganglion in Monoiodoacetate-Induced Rats of Osteoarthritis
}

\section{Wenwen Zhou}

The Affiliated Hospital of Qingdao University

\section{Lin Wang}

The Affiliated Hospital of Qingdao University

\section{Qilong Cao}

Haier Group

Xinhe LI

The Affiliated Hospital of Qingdao University

Yue Hu

Qingdao University

Juan Li

The Affiliated Hospital of Qingdao University

Tieshan Li ( $\sim$ tieshanl@126.com)

The Affiliated Hospital of Qingdao University

\section{Research Article}

Keywords: mesenchymal stem cell derived exosomes, dorsal root ganglion, Osteoarthritis, cartilage, neuropathic pain, Pain relief

Posted Date: May 24th, 2021

DOI: https://doi.org/10.21203/rs.3.rs-535017/v1

License: (c) (1) This work is licensed under a Creative Commons Attribution 4.0 International License. Read Full License 


\section{Intraarticular injection of different doses of mesenchymal stem cell derived exosomes reduces ATF-3 expression in the dorsal root ganglion in monoiodoacetate-induced rats of osteoarthritis}

\author{
Sample of title note
}

Wenwen $\mathrm{Zhou}^{1}$, Lin Wang ${ }^{1}$, Qilong $\mathrm{Cao}^{2 \dagger}$, Xinhe $\mathrm{Li}^{1 \dagger}$, Yue $\mathrm{Hu}^{3}$, Juan $\mathrm{Li}^{1}$ and Tieshan $\mathrm{Li}^{1 *}$

\begin{abstract}
Background: This study aims to evaluate the therapeutic effect of intra-articular injection of different doses of exosomes derived from mesenchymal stem cells(MSC) and the effect on nerve and cartilage repair in a monoiodoacetate (MIA) model of knee osteoarthritis $(\mathrm{OA})$ in rats.
\end{abstract}

Methods: The pain rat model was established by injection of sodium monoiodate (MIA) into the knee joint of the rats, the knee joint and dorsal root ganglion (DRG) of rats were collected for histologic analyses. For pain assessment, On 1 day before MIA injection, 7, 14 days after MIA injection and 7, 14,28 days after Exosome injection, paw withdrawal threshold (PWT) and paw withdrawal latency (PWL) was measured. Articular cartilage were assessed on HE by ORASI grade and the expression of ATF-3 and GAP-43 in dorsal root ganglion (DRG) using immunohistochemistry and western blotting in MIA-induced rats.

Results: In our study, exosome significantly improved PWT and PWL value with a dose-dependent manner on 7, 14, and 28 days after intra-articular Exosome compared with the MIA group. Exosome injection therapy also has a repairing effect on cartilage on 28 days after intra-articular Exosome compared with the MIA group. Moreover, exosome treatment significantly upregulated GAP-43 protein and downregulated ATF-3 protein in the DRG with a dose-dependent manner of the OA rat.

Conclusion: Intraarticular injection of different doses of mesenchymal stem cell derived exosomes in MIA-induced rats osteoarthritis, the analgesic effect of exosome was dose-dependent. Moreover, the repair of nerve by exosomes is earlier than that of cartilage.

Keywords: mesenchymal stem cell derived exosomes; dorsal root ganglion; Osteoarthritis; cartilage; neuropathic pain; Pain relief

\section{Backgroung}

Osteoarthritis (OA) [1] is one of the most ubiquitous joint disease, resulting in pain, stiffness, and disability. OA of the knee, hand, or hip affects $20-30 \%$ of adults in different populations and is increasing in prevalence[2]. In the US alone, 27 million adults are affected by this disease, which is expected to reach 67 million by $2030[3,4]$,Seriously affects people's daily life. Pain is the most common and promi- 
nent clinical feature of $\mathrm{OA}$, and it is also the major reason for patients to visit a physician[5]. In patients over 60 years old, the incidence of knee pain secondary to osteoarthritis (OA) is $12 \%[5]$, Which creating a signifcant individual and societal burden.

Currently, the pathogenesis of OA has not been fully clarified, there is a lack of specific treatment that slow, halt, or reverse the progression of joint damage in OA - At present, Traditional conservative treatment including pharmacologic agents( non-steroidal anti-inflammatory drugs (NSAIDs), opioid analgesics and tramadol), intra-articular injection( steroids, viscosupplementation and hyaluronic acid), physical therapy and other means, but these means have limited efficacy and certain side effects, and only aiming for pain reduction and symptom control, rather than reversing the process of $\mathrm{OA}[6,7,8]$. For more serious cases, Total knee arthroplasty (TKA) is the last choice, Although TKA is more effective, it is not without significant complication, as many as $2 \%$ of patients will have significant complications, such as death, pulmonary embolism and infection, and this method is not suitable for young patients with a lot of activity. What's more, up to $20 \%$ of patients will continue to have knee pain $[9,10]$.

Recent research emphasize that OA is a heterogeneous disease of the whole joint. In the early stage, osteoarthritis may be predominantly bone-focused, cartilagefocused or inflammation-focused[11, 12]. However, in advanced osteoarthritis, different pathological processes may lead to similar end-stage phenotypes ,that is, neuropathic pain $[12,13]$. Neuropathic pain is defined as pain caused by a lesion or disease of the somatosensory system. some reports and reviews have concluded that a proportion of OA patients suffer from neuropathic pain. E.g., Hochman et al.[14] reported that $34 \%$ of the patients in their study suffered from neuropathic pain . Therefore, In order to postpone the TKA operation as much as possible, looking for a new treatment which can block neuropathy pain and produce rapid and long-lasting analgesia effects is sorely needed.

Recently, mesenchymal stem cells (MSCs)have been widely applied to the treatment of pain as an alternative or promising treatment for severe osteoarthritis, neuropathic pain, and intractable musculoskeletal pain which are ineffective to traditional drugs[15]. In several preclinical studies, have confirmed that MSC have a role in neuroprotective and axonal growth stimulation in various models of nervous system injury[16, 17], In a number of preclinical and clinical studies, intraarticular injections (IA) of MSCs can relieve pain and improve function[18]. However, almost all MSCs have their own limitations, such as high requirements for isolation, collection and transportation, high requirements for the age of the donor, and limited proliferation capacity in vitro. In addition, the number and repair ability of stem cells will decrease with the normal metabolism of human body, which will eventually affect the activity and curative effect of stem cells. Therefore, it has become the focus of attention in recent years to find a kind of stem cell substitute which is easy to collect, low immunogenicity, lack endogenous tumor-formation potential, in vivo stability, and high delivery efficiency.

With the in-depth study of stem cells, accumulating evidence has show that many of the therapy properties previously credited to MSCs should be attributed to the secreted exosomes(Exo)[19, 20]. Exosomes are extracellular vesicles containing a 
variety of active substances, which play a key role in intercellular and intracellular communication. At present, several pre-clinical studies have confirmed that exosomes derived from MSCs can relieve pain and improve function. However, its analgesic mechanism has not been clarified, and there is controversy about whether cartilage is repaired[21, 22]. In addition, there is no unified standard for the treatment of OA with exosomes. Therefore, this study aims to establish a monoiodoacetate (MIA) model of knee osteoarthritis in rats to evaluate the therapeutic effect of intra-articular injection of different doses of exosomes derived from MSCs, and observe whether there is a dose-dependent effect and the mechanism of analgesic effect.

\section{Materials and Methods}

\section{Animals}

Adult male Sprague-Dawley (SD) rats weighing 170-200 g were provided by the Experimental Animal Center of Qingdao University, These animals are kept in a room at $23^{\circ} \mathrm{C} \pm 1{ }^{\circ} \mathrm{C}$, with light and dark cycles of day and night $(12 \mathrm{~h}$ light $/ 12 \mathrm{~h}$ dark), and free access to water and food.The rats were accustomed to the testing facility at least 5 days before the collection date. The breeding conditions and experimental procedures comply with the regulations approved by the Animal Care and Use Committee of Qingdao University. Make every effort to minimize animal suffering.

Drug

Animal model

The pain rat model was induced with an injection of $4 \mathrm{mg} / 50 \mathrm{ul}$ sodium monoiodate (MIA) into the knee joint cavity of the left(ipsilateral) leg of the rats by microsyringe under light anesthesia with $5 \%$ isoflurane,Similarly, the control group were injected with 50ul normal saline.

Administration of Exosomes

On day 14 after the injection of MIA, rats were anesthetized by $5 \%$ isoflurane. A 100ul soution containing Exosome (40ug/100ul,100ug/100ul,200ug/100ul, Qingdao Haier Biotech Co.) or an equivalent volume of saline was injected into the unilateral knee articular cavity of the rats. The concentration of exosome solution was chosen based on a previous experimental study[21, 23]. ninety male SD rats were randomly divided into 5 groups with 18 in each group:(1) the sham group (control rats injected with $100 \mathrm{pl}$ saline), (2) the MIA group (MIA rats injected with 100ul saline),(3) the 40ug/100ul exosome group (MIA rats injected with 40ug/100ul exosome/100ul),(4)The 100ug/100ul exosome group (MIA rats injected with100ug/100ul exosome/100ul), and (5) the 200ug/100ul exosome group (MIA rats injected with 200ug/100ul exosome/100ul).

\section{Behavioral tests}

mechanical allodynia

According to the method reported in Chaplan (1994)[24], the 50\% withdrawal threshold (PWT) was determined by von Frey filament to evaluate the mechanical allodynia. The rats were placed on a clear plexiglass lattice platform enclosed 
with mesh and allowed to habituate for $30 \mathrm{~min}$, The observer was blinded to the treatment groups, On 1 day before MIA injection, 7, 14 days after MIA injection and 7, 14,28 days after Exosome injection, PWT was measured in an increasing order of force $(0.4 \mathrm{~g}-15.0 \mathrm{~g})$ to the plantar surface perpendicular to the paw. And always from $2.0 \mathrm{~g}$ fowling up and down method, and then the pressure was gradually increased until the foot was quick withdrawaled or licked. The test was continued 4-5 times from the time when the reaction occurred, each stimulation interval was $1 \mathrm{~min}$, and then $50 \%$ PWT was calculated by special formula.

\section{Thermal hyperalgesia}

According to $\mathrm{Yu}$ et al., the paw withdrawal latency (PWL) was evaluated by hot plate, which was used to indicate the thermal pain threshold[25]. The rats were placed on the hot plate $\left(50 \pm 0.5{ }^{\circ} \mathrm{C}\right)$, The observer was blinded to the treatment groups, On 1 day before MIA injection, 7, 14 days after MIA injection and 7, 14,28 days after Exosome injection, the time that the rat jumped or licked its hind paws was used as the PWL, Once the rats make a positive reaction, immediately remove the rats from the hot plate, so as not to cause damage to the rats and affect the follow-up experimental results. The average value was taken after three tests with an interval of 15 minutes.

Hematoxylin and eosin (HE) staining for the cartilage

For histopathological analysis, On 1 day before MIA injection, 7, 14 days after MIA injection and 7,14,28 days after Exosome injection, Three rats in each group were anesthetized by intraperitoneal injection of $10 \%$ chloral hydrate, rat knee joints were fifixed in paraformaldehyde at $4{ }^{\circ} \mathrm{C}$ for $6-8 \mathrm{~h}$, and decalcifified using ethylene diamine tetra acetic acid (EDTA) for 2-4 weeks.After being embedded in paraffin and dehydrated, Paraffifin sections (5-10mm thickness) of the knee joint were stained with haematoxylin and eosin (HE). After sealing, The Osteoarthritis Research Society International (OARSI) score was used for assessment[26], the slices were observed under microscope and ImageJ 1.36 software system was used for image analysis to observe the degeneration and repair of ankle cartilage in each group.The observer was blinded to the treatment groups.

\section{ATF-3and GAP-43 expression detection using western blot analysis}

For protein detection of ATF-3 and GAP-43, On 1 day before MIA injection, 7, 14 days after MIA injection and 7, 14,28 days after Exosome injection, L3- L5 DRG tissues were homogenized in ice-cold lysis buffer (Sigma, USA). The lysates were spun at $12,000 \mathrm{~g}$ for $15 \mathrm{~min}$ at $4{ }^{\circ} \mathrm{C}$ and supernatants were used for western blotting. Equal amounts of denatured protein (40ug)were separated through 12\% sodium dodecyl sulphate polyacrylamide (SDS-PAGE) (Sigma, USA) and then transferred onto Polyvinylidene Fluoride(PVDF) membranes (290 mA, 75 min). The membranes were blocked with $5 \%$ non-fat dry milk in TBST for 2 hour at room temperature to block nonspecific binding sites. The membranes were then incubated with antibodies to ATF-3(diluted 1:600, Abcam),GAP-43(diluted 1:5000, Bioss) or GAPDH(diluted 1:5000, Abcam) overnight at $4^{\circ} \mathrm{C}$. After 3 washes in TBST for $10 \mathrm{~min}$, the membranes were incubated with goat anti-rabbit IgG (diluted 1:5000,Abcam ) for 75 
min at room temperature. The chemiluminescent ECL reagent (Millipore, USA) was used to visualize the protein bands. Band densities were analyzed using the Image J software.

The expression of ATF-3 in DRG was detected by immunohistochemistry For detection of ATF-3, On 1 day before MIA injection, 28 days after MIA injection and 28 days after Exosome injection, Three rats in each group were anesthetized by intraperitoneal injection of $10 \%$ chloral hydrate, and then sequentially perfused with saline and $4 \%$ paraformaldehyde ( $\mathrm{pH} 7.4$ ). Subsequently, the L5 DRG was placed in the $4 \%$ paraformaldehyde for $24 \mathrm{~h}$ then ethanol gradient dehydration, after being embedded in paraffin, sections were cut transversely at a 5 um thickness, The slides were incubated with $3 \% \mathrm{H} 2 \mathrm{O} 2$ at room temperature for 30 min to eliminate endogenous peroxidase activity and then treated for 10 minutes with $0.01 \mathrm{M}$ sodium citrate buffer ( $\mathrm{pH} \mathrm{6.0)}$ for antigen retrieval. The slides washed in PBS for 5 min and then blocked with $5 \%$ normal goat serum containing $0.1 \%$ Triton X-100 in PBS for 30 min and incubated overnight with antibodies to ATF-3(diluted 1:100, Abcam) . After 3 washes in TBST for $10 \mathrm{~min}$, the slides were incubated with goat anti-rabbit IgG (diluted 1:5000,Abcam ) for 30 min at room temperature. Add the DAB color developing solution, take the brown yellow color as positive color, then rinse the slices with tap water for 30 minutes, re dye with hematoxylin, then dehydrate with alcohol, gradient dry, transparent with xylene for 2 times, finally seal and observe the results under optical microscope.

\section{Statistical analysis}

Statistically significant difference analysis was carried out using SPSS 26 software . All data were expressed as the mean \pm standard error of the mean (SEM). For pain behavioral tests (PWT and PWL), two-way repeated measures analysis of variance (RMANOVA) were used for analyzing the effects of tested doses of Exosome on pain behaviors at different time points. measurements of western blot, immunohistochemistry as well as HE staining analysis were performed using one-way ANOVA to investigate the differences between the groups. Statistically significant level was set at $\mathrm{p}<0.05$.

\section{Results}

Pain behavioral effect of Exosome on MIA-induced rats

There were no significant differences in pain-related behaviors (PWT and PWL) among the five groups before MIA injection. Compared with the baseline and the sham control,all animals showed significant decreases in PWT (Fig. 1A, P<0.001) and PWL (Fig. 1B, P<0.001)from 1 to 42 days after MIA injection. 14 days after MIA injection, Exosome or Saline were injected into the knee articular ipsilateral to the MIA rats.Compared with the MIA group, Exosome significantly increased PWT to mechanical stimulation with a dose-dependent manner as the threshold reversal was more obvious at a dose of $100 \mathrm{ug} / 100 \mathrm{uL}, 200 \mathrm{ug} / 100 \mathrm{uL}$ than $40 \mathrm{ug} / 100 \mathrm{uL}$ on 7,14 , and 28 days after intra-articular Exosome(Fig. 1A). In a similar way, the PWL was also significantly reversed to thermal stimulation by the injection of Exosome(Fig. 1B). what 's more, there was no difference in 100ug/100uL compared with the 200ug/100uL groups in the PWT and PWL . 
Exosome caused cartilage repair in late stage on MIA-induced rats

On 1 day before MIA injection, 7, 14 days after MIA injection and 7, 14,28 days after Exosome injection, the knee joint specimens of animals were collected for HE (Fig. $2 \mathrm{~A}$ ). The staining showed that in the sham group, the cartilage surface is smooth , no enlargement/distortion of chondrons and no proliferative changes of chondrocytes are observed.The surface discontinuity accompanied by cell proliferation, and cell death, the matrix fifibrillation extends vertically downward into the mid zone and cartilage matrix loss is observed in the MIA group . Fibrocartilage repair was observed at a dose of $40 \mathrm{ug} / 100 \mathrm{uL}, 100 \mathrm{ug} / 100 \mathrm{uL}$ and $200 \mathrm{ug} / 100 \mathrm{uL}$ on 28 days after intra-articular Exosome. Compared with the sham control,all animals showed significant statistical difference in OARSI grade (Fig. 2B, $\mathrm{P}<0.001$ ) from 1 to 42 days after MIA injection. There was no difference in MIA group compared with the Exosome groups on 7,14 days after intra-articular Exosome(Fig. 2B), Compared to the MIA group,all Exosome groups showed significant statistical difference in OARSI grade (Fig. 2B, $\mathrm{P}<0.001$ ) on 28 days after Exosome injection.what 's more , there was no difference between group in $40 \mathrm{ug} / 100 \mathrm{uL}$ and group in $100 \mathrm{ug} / 100 \mathrm{uL}$ and groups in $200 \mathrm{ug} / 100 \mathrm{uL}$.

\section{Effects of exosome on expression of ATF-3 and GAP-43 protein in DRG on MIA-induced rats}

The results of Western blotting were shown in Figure 3. Compared with baseline values, the protein expression of ATF-3 and GAP-43 was significantly increased on the 14th day after MIA injection $(\mathrm{P} i<0.001$ ), (Fig. 3A,3B) but there was no significant difference between the sham group and MIA group on the 7th day after MIA injection.

Compared with the sham control, all animals showed significant statistical difference in ATF-3 and GAP-43 experssion (Fig. 3C,3D) from 7 to 28 days after Exosome injection. Compared to the MIA group, Exosome caused the levels of ATF-3 and GAP-43 protein in the DRG in a dose-dependent manner (Fig. 3C and D). Compared with the MIA group, Exosome significantly decreased ATF-3 expression at a dose of $100 \mathrm{ug} / 100 \mathrm{uL}, 200 \mathrm{ug} / 100 \mathrm{uL}$ than $40 \mathrm{ug} / 100 \mathrm{uL}$ on 7,14 , and 28 days after intra-articular Exosome. In a similar way, the GAP-43 expression was also significantly increased at a dose of $100 \mathrm{ug} / 100 \mathrm{uL}, 200 \mathrm{ug} / 100 \mathrm{uL}$ than $40 \mathrm{ug} / 100 \mathrm{uL}$ on 7 , 14 , and 28 days by the injection of Exosome. what 's more, there was no difference in $100 \mathrm{ug} / 100 \mathrm{uL}$ compared with the $200 \mathrm{ug} / 100 \mathrm{uL}$ groups in the ATF-3 and GAP-43 expression.

\section{Effects of exosome on expression of ATF-3 protein by immunohistochemistry in DRG on MIA-induced rats}

Compared with the sham control, all animals showed significant statistical difference in ATF-3 -positive neurons(Fig. 4A) on 28 days after Exosome injection. Compared with the MIA group, Exosome significantly decreased ATF-3-positive neurons at a dose of $40 \mathrm{ug} / 100 \mathrm{uL}, 100 \mathrm{ug} / 100 \mathrm{uL}$ and $200 \mathrm{ug} / 100 \mathrm{uL}$ on 28 days after intra-articular Exosome. what 's more, there was no difference in $100 \mathrm{ug} / 100 \mathrm{uL}$ compared with the $200 \mathrm{ug} / 100 \mathrm{uL}$ groups in the ATF-3 positive neurons(Fig. 4B). 


\section{Discussion}

The aim of the present study was to evaluate the therapeutic effects of exosomes secreted by mesenchymal stem cells injected directly into the knee joints of MIA-induced OA rats. For this purpose, we conducted the following procedures. First, Pain-related behavior was assessed on mechanical allodynia (presented by PWT) and thermal hyperalgesia (presented by PWL) in MIA-induced rats. Second, articular cartilage was assessed on HE by ORASI grade in MIA-induced rats .Third,evaluation of pain of OA using immunohistochemistry and western blotting of ATF-3 ,GAP-43 in DRG. The results demonstrated that direct intraarticular injection of Exosomes into the joints of knee OA model induced by MIA resulted in cartilage repair and alleviate the pain. To our best knowledge, This is the first report that compares the therapeutic effects of direct intraarticular injection of different doses of Exosome on cartilage degeneration and analgesic effect in MIA-induced knee OA.

Although recently it has been confirmed that the efficacy of MSC-derived exosomes on therapeutic effect in the animal models of $\mathrm{OA}[27,28,29]$, there are few studies on the therapeutic effect of MSC-derived exosomes on mechanism of pain relief. Previous studies have shown that both intraarticular injection of MIA and partial medial meniscus resection of the knee joint OA model can induce histological changes and pain-related behavioral changes in the knee joint, but the OA model induced by MIA is more consistent with the pain characteristics of clinical symptoms[30]. In the MIA of OA, the most frequently used dose is 1, 2 or 4 $\mathrm{mg}[31,30,32]$, with the model usually evaluated within 14 days post-induction. It has been proved that high-dose MIA induced osteoarthritis pain originate from inflammation caused by cytokines, which leads to progressive, chronic neuronal damage that may cause neuropathic pain. Moreover, the low-level inflammation caused by MIA usually resolves fully by day $7[33,34]$.

In this study, we used the methods of pain behavior and molecular biology to explore the specific relationship between the analgesic effect and different doses of exosomes. The results of pain behavior showed that intra-articular administration of Exosome could significantly reduce nociceptive behaviors in a dose-dependent manner (Fig. 1A, 1B). Our dates showed that Exosome had antinociceptive effect on mechanical allodynia and thermal hyperalgesia for a long time, rising within 7 days and maintained a higher level 28 days post intra-articular administration at dose of $100 \mathrm{ug} / 100 \mathrm{uL}$ or $200 \mathrm{ug} / 100 \mathrm{uL}$, A dose of 40ug/100uL Exosome rising within 14 days and maintained a higher level 28 days post intra-articular administration. in addition, Our study showed that PWT and PWL were significantly lower in the OA group than in the sham group after MIA injection, indicating that hyperalgesia and allodynia are involved in the pathogenesis of OA pain, which is consistent with Mapp et al.'s findings[35]. Hyperalgesia and abnormal are the characteristic manifestations of neuropathic pain and the results of central and peripheral sensitization of neuropathic pain.

Dorsal root ganglion (DRG) neuronal injury is an important cause of neuropathic pain and pain sensitization. DRG belongs to the peripheral sensory ganglia. DRG neurons are the primary afferent neurons for pain, which have the functions of transmitting and regulating body sensation, receiving and conducting nociception. 
In the process of pain production, the dorsal root ganglion, as the primary neuron of pain afferent, plays an important role in the pain mechanism. There are research findings showing the increased expression of ATF-3 in DRG neurons by using rat MIA-induced OA models[36]. ATF3 is reported to be a selective marker of nerve injury[37]. GAP-43 is a marker of regenerating nerve fibers[38]. ATF3 is not thought be expressed during inflammation[39, ?], thus the increased expression of ATF3 in DRG neurons suggests the evidence of gradually progressive nerve injuries. To clarify their association with nerve injury, evaluating the expression of GAP-43 and ATF3 at the level of the DRG may be useful because these molecules are elevated in DRG neurons with regenerating axon fibers after nerve injury, which may indicate the pathogenesis of neuropathic pain[40]. Simultaneously, the significant gradual increase the expression of ATF3 and GAP-43 in DRG neurons implies a restoration process of the injured nerves in addition to the nerve injuries. Our dates showed that Exosome had upgrade the GAP-43 expression and degrade the ATF-3 expression for a long time, Exosome significantly decreased ATF-3 expression at a dose of $100 \mathrm{ug} / 100 \mathrm{uL}, 200 \mathrm{ug} / 100 \mathrm{uL}$ than $40 \mathrm{ug} / 100 \mathrm{uL}$ on 7,14 , and 28 days after intra-articular Exosome. In a similar way, the GAP-43 expression was also significantly increased at a dose of $100 \mathrm{ug} / 100 \mathrm{uL}, 200 \mathrm{ug} / 100 \mathrm{uL}$ than $40 \mathrm{ug} / 100 \mathrm{uL}$ on 7 , 14, and 28 days by the injection of Exosome. These experimental results revealed that exosomes can reduce nerve damage and promote nerve repair. Interestingly, the expression of ATF-3 and GAP-43 increased on 14 days after MIA injection. It shows that progressive nerve damage and regeneration may be the result of cartilage degradation; the physical wearing of cartilage leads to exposure and degradation of subchondral bone, where sensory nerve ingrowth and pain-related mediators increase $[41, ?]$. Subsequently, the exposed nerve endings may become physically injured, which accelerates nerve ingrowth into the subchondral bone.This may result in a temporary increase in the expression of ATF3 and GAP43.

In this study, we used the Hematoxylin and eosin (HE) staining to assessed the articular cartilage repair on different doses of exosomes. The results of histopathology showed that Exosome could promote cartilage repair at dose of $40 \mathrm{ug} / 100 \mathrm{uL}, 100 \mathrm{ug} / 100 \mathrm{uL}$ or $200 \mathrm{ug} / 100 \mathrm{uL}$ on 28 days after intra-articular Exosome, but not at 7 and 14 days .Our study showed that exosome can caused cartilage repair which is consistent with Lei et al.'s findings[21]. As far as we know, although the pathogenesis of osteoarthritis begins with cartilage degeneration and then leads to nerve damage, our experimental results show that the repair of nerve by exosomes is earlier than that of cartilage, which indicate nerve repair plays an important role in the early stage of OA pain relief.

There are still some limitations to this study.First, MIA-induced OA is a chemically induced model.The signal seen in the MIA model could be the result of a direct action of MIA on the peripheral nerves running adjacent to the knee, To clarify the details, further investigation with other nonchemically induced pathological models, such as partial medial meniscectomy, should be conducted. Second, although we observed exosome treatment effectively alleviated articular cartilage injury and pain in OA rats, we did not further investigate the molecular mechanism underlying the upstream signaling molecule. In addition, the therapeutic effect of MSC-derived exosomes remains to be validated in a clinical trial. All these limitations should be addressed in the future study. 


\section{Conclusions}

In summary, our results demonstrated that 1).Intraarticular injection of different doses of mesenchymal stem cell derived exosomes in MIA-induced osteoarthritis of rats, the analgesic effect of exo was dose-dependent. Within a certain dose range, the analgesic effect gradually increases with the increase of the dose. But beyond this dose range, even if the dose is increased, the analgesic effect will not change. 2). Exo injection therapy has a repairing effect on cartilage, but early pain relief may not be related to cartilage repair, nerve repair plays an important role in OA pain relief. which indicate a new choice for clinical treatment of pain in patients with OA.

\section{Acknowledgements}

Not applicable.

Funding

This work was supported by the Qingdao Technology Bureau (No.13-1-3-34-nsh) and the Shandong Natural Science Foundation (ZR2018MH031).

\section{Abbreviations}

OA: Osteoarthritis; MSCs: Mesenchymal stem cells; H\&E: Hematoxylin and eosin; OARSI: Osteoarthritis Research Society International; IHC: Immunohistochemistry; PWT: Paw withdrawal threshold; PWL: Paw withdrawal latency; DRG: Dorsal root ganglion; MIA:monoiodoacetate;ATF-3: Activating Transcription Factor 3; GAP-43:growth associated protein 43

Availability of data and materials

All the data and materials were presented in the main paper

Ethics approval and consent to participate

All experimental protocols were approved by Animal Care and Use Committee of Qingdao University and carried out in accordance with the guidelines of the International Association for the Study of Pain(Zimmermann, 1983).

Competing interests

The authors declare that they have no competing interests.

Consent for publication

Not applicable

Authors' contributions

1, Wenwen Zhou and Tieshan Li: designed experiments 2, Wenwen Zhou, Xinhe Li and Qi long Cao: carried out experiments 3, Lin Wang: analyzed experimental results 4, Wenwen Zhou: wrote (original draft preparation) 5 , Wenwen Zhou, Yue Hu and Juan Li: wrote (review and editing) 6, Tieshan Li: funding acquisition

\section{Author details}

${ }^{1}$ Department of Rehabilitation Medicine, The Affiliated Hospital of Qingdao University, QingDao, China.

${ }^{2}$ Department of Qingdao Haier Biotech Co,Ltd,, QingDao, China. ${ }^{3}$ Department of Rehabilitation Medicine, Qingdao University, QingDao, China.

\section{References}

1. The global burden of hip and knee osteoarthritis: estimates from the global burden of disease 2010 study. Annals of the Rheumatic Diseases 73(7), 1323 (2014)

2. Neogi, T., Zhang, Y.: Epidemiology of oa. Rheumatic Diseases Clinics of North America 39(1), 1 (2013)

3. Hootman, J.M., Helmick, C.G.: Projections of us prevalence of arthritis and associated activity limitations. Arthritis \& Rheumatology 54(1), 226-229 (2014)

4. Hootman, J.M., Helmick, C.G., Barbour, K.E., Theis, K.A., Boring, M.A.: Updated projected prevalence of self-reported doctor-diagnosed arthritis and arthritis-attributable activity limitation among us adults, 2015-2040. Arthritis Rheumatol 68(7), 1582-1587 (2016)

5. Neogi, T.: The epidemiology and impact of pain in osteoarthritis. Osteoarthritis and Cartilage 21(9), 1145-1153 (2013)

6. Hermann, W., Lambova, S., Muller-Ladner, U.: Current treatment options for osteoarthritis. Current Rheumatology Reviews (2018)

7. Ding, C.: Do nsaids affect the progression of osteoarthritis? Inflammation 26(3), 139-142 (2002)

8. Oliviero, F., Ramonda, R., Punzi, L.: New horizons in osteoarthritis. Schweizerische medizinische Wochenschrift 140, 13098 (2010)

9. Singh, J.A., Kundukulam, J., Riddle, D.L., Strand, V., Tugwell, P.: Early postoperative mortality following joint arthroplasty: A systematic review. Journal of Rheumatology 38(7), 1507-1513 (2011)

10. Wylde, V., Hewlett, S., Learmonth, I.D., Dieppe, P.: Persistent pain after joint replacement: prevalence, sensory qualities, and postoperative determinants. Pain 152(3), 566-572 (2011) 
11. Karsdal, M.A., Michaelis, M., Ladel, C., Siebuhr, A.S., Bihlet, A.R., Andersen, J.R., Guehring, H., Christiansen, C., Bay-Jensen, .A., Kraus, V.B.: Disease-modifying treatments for osteoarthritis (dmoads) of the knee and hip: lessons learned from failures and opportunities for the future. Osteoarthritis and Cartilage, 2013-2021 (2016)

12. Siebuhr, A., Bay-Jensen, A., Jordan, J., Kjelgaard-Petersen, C., Christiansen, C., Abramson, S., Attur, M., Berenbaum, .F., Kraus, V., Karsdal, M.: Inflammation (or synovitis)-driven osteoarthritis: an opportunity for personalizing prognosis and treatment? Scandinavian Journal of Rheumatology, 87-98 (2016)

13. Dell'Isola, A., Allan, R., Smith, S.L., Marreiros, S., Steultjens, M.: Identification of clinical phenotypes in knee osteoarthritis: a systematic review of the literature. BMC Musculoskeletal Disorders 17(1), 425 (2016)

14. Hofmann, G.O., Marticke, J., Grossstück, R., Hoffmann, M., Lange, M., Plettenberg, H., Braunschweig, R., Schilling, O., Kaden, I., Spahn, G.: Detection and evaluation of initial cartilage pathology in man: A comparison between mrt, arthroscopy and near-infrared spectroscopy ( $\mathrm{nir}$ ) in their relation to initial knee pain. Pathophysiology 17(1), 1-8 (2010)

15. Han, Y., Kim, K., Abdi, S., Kim, T.K.: Stem cell therapy in pain medicine. The Korean Journal of Pain 32(4) (2019)

16. Abel, Torres-Espín, Dora, Luz, Corona-Quintanilla, Joaquim, Forés, Ilary, Allodi, Francisco: Neuroprotection and axonal regeneration after lumbar ventral root avulsion by re-implantation and mesenchymal stem cells transplant combined therapy. Neurotherapeutics 10(2), 354-368 (2013)

17. Cao, Q., Benton, R.L., Whittemore, S.R.: Stem cell repair of central nervous system injury. Journal of Neuroence Research 68(5), 501-510 (2002)

18. Freitag, J., Kenihan, M.A.: Mesenchymal stem cell therapy in osteoarthritis and regenerative medicine. Current Sports Medicine Reports 17(12), 441-443 (2018)

19. Basu, J., Ludlow, J.W.: Exosomes for regeneration, rejuvenation, and repair (2018)

20. Baglio, S.R., Pegtel, D.M., Baldini, N.: Mesenchymal stem cell secreted vesicles provide novel opportunities in (stem) cell-free therapy. Frontiers in Physiology 3 (2012)

21. He, L., He, T., Xing, J., Zhou, Q., Rong, L.: Bone marrow mesenchymal stem cell-derived exosomes protect cartilage damage and relieve knee osteoarthritis pain in a rat model of osteoarthritis. Stem Cell Research and Therapy 11(1) (2020)

22. Sakamoto, T., Miyazaki, T., Watanabe, S., Ai, T., Matsumine, A.: Intraarticular injection of processed lipoaspirate cells has anti-inflammatory and analgesic effects but does not improve degenerative changes in murine monoiodoacetate-induced osteoarthritis. BMC Musculoskeletal Disorders 20(1) (2019)

23. Si, H., Tjio, C., Wong, J., Wong, K.L., Wei, S.T.: Mesenchymal stem cell exosomes for cartilage regeneration: A systematic review of preclinical in vivo studies. Tissue Engineering Part B Reviews (2020)

24. Chaplan, S.R., Bach, F.W., Pogrel, J.W., Chung, J.M., Yaksh, T.L.: Quantitative assessment of tactile allodynia in the rat paw. Journal of Neuroscience Methods 53(1), 55-63 (1994)

25. Lu, Y., Yang, F., Hao, L., Liu, F.Y., Han, J.S., Xing. ., G.: The role of trpv1 in different subtypes of dorsal root ganglion neurons in rat chronic inflammatory nociception induced by complete freund's adjuvant. Molecular Pain 4(1), 61 (2008)

26. CS., Carlson, N., Gerwin, A., M., Bendele, S., Glasson: The oarsi histopathology initiative - recommendations for histological assessments of osteoarthritis in the rat. Osteoarthritis and Cartilage (2010)

27. Yubao, Liu, Rui, Zou, Zhen, Wang, Chuanyang, Wen, Fan, Zhang: Exosomal klf3-as1 from hmscs promoted cartilage repair and chondrocyte proliferation in osteoarthritis. Biochemical Journal (2018)

28. Yafei, Wang, Dongsheng, Yu, Zhiming, Liu, Fang, Zhou, Jun, Dai: Exosomes from embryonic mesenchymal stem cells alleviate osteoarthritis through balancing synthesis and degradation of cartilage extracellular matrix. Stem Cell Research and Therapy 8(1), 189 (2017)

29. Zhang, S., Teo, K., Chuah, S.J., Lai, R.C., Lim, S.K., Toh, W.S.: Msc exosomes alleviate temporomandibular joint osteoarthritis by attenuating inflammation and restoring matrix homeostasis. Biomaterials 200, 35-47 (2019)

30. Fernihough, J., Gentry, C., Malcangio, M., Fox, A., Rediske, J., Pellas, T., Kidd, B., Bevan, S., Winter, J.: Pain related behaviour in two models of osteoarthritis in the rat knee. Pain 112(1-2), 83-93 (2004)

31. Janusz, M.J., Hookfin, E.B., Heitmeyer, S.A., Woessner, J.F., Freemont, A.J., Hoyland, J.A., Brown, K.K., Hsieh, L.C., Almstead, N.G., De, B.: Moderation of iodoacetate-induced experimental osteoarthritis in rats by matrix metalloproteinase inhibitors. Osteoarthritis and Cartilage 9(8), 751-760 (2001)

32. Pomonis, J.D., Boulet, J.M., Gottshall, S.L., Phillips, S., Sellers, R., Bunton, T., Walker, K.: Development and pharmacological characterization of a rat model of osteoarthritis pain. Pain 114(3), 339-346 (2005)

33. Clements, K.M., Ball, A.D., Jones, H.B., Brinckmann, S., Read, S.J., Murray, F.: Cellular and histopathological changes in the infrapatellar fat pad in the monoiodoacetate model of osteoarthritis pain. Osteoarthritis and Cartilage 17(6), 805-812 (2009)

34. Jacobs, B.Y., Kloefkorn, H.E., Allen, K.D.: Gait analysis methods for rodent models of osteoarthritis. Current Pain and Headache Reports 18(10) (2014)

35. Mapp, P.I., Sagar, D.R., Ashraf, S., Burston, J.J., Walsh, D.A.: Differences in structural and pain phenotypes in the sodium monoiodoacetate and meniscal transection models of osteoarthritis. Osteoarthritis and Cartilage 21(9), 1336-1345 (2013)

36. Ivanavicius, S.P., Ball, A.D., Heapy, C.G., Westwood, F.R., Murray, F., Read, S.J.: Structural pathology in a rodent model of osteoarthritis is associated with neuropathic pain: increased expression of atf- 3 and pharmacological characterisation. Pain 128(3), 272-282 (2007)

37. Tsujino, H., Kondo, E., Fukuoka, T., Yi, D., Tokunaga, A., Miki, K., Yonenobu, K., Ochi, T., Noguchi, K.: Activating transcription factor 3 (atf3) induction by axotomy in sensory and motoneurons: A novel neuronal marker of nerve injury. Molecular and Cellular Neuroscience 15(2), 170-182 (2000)

38. Antigen-induced arthritis in rats is associated with increased growth-associated protein43-positive intraepidermal nerve fibres remote from the joint. Arthritis Research and Therapy 17(1), 299 (2015)

39. Averill, S., Michael, G.J., Shortland, P.J., Leavesley, R.C., Priestley, J.V.: Ngf and gdnf ameliorate the increase in atf3 expression which occurs in dorsal root ganglion cells in response to peripheral nerve injury. European 
Journal of Neuroscience 19(6), 1437-1445 (2015)

40. Orita, S., Ishikawa, T., Miyagi, M., Ochiai, N., Inoue, G., Eguchi, Y., Kamoda, H., Arai, G., Toyone, T., Aoki, Y.: Pain-related sensory innervation in monoiodoacetate-induced osteoarthritis in rat knees that gradually develops neuronal injury in addition to inflammatory pain. Bmc Musculoskeletal Disorders 12(1), 134 (2011)

41. Ogino, S., Sasho, T., Nakagawa, K., Suzuki, M., Yamaguchi, S., Higashi, M., Takahashi, K., Moriya, H.: Detection of pain-related molecules in the subchondral bone of osteoarthritic knees. Clinical Rheumatology 28(12), 1395-1402 (2009)

Figures

Figure 1 Pain-related behavior was assessed in Sprague-Dawley rats. Effects of tested doses of Exosome (40ug/100uL,100ug/100uL, 200ug/100uL) on mechanical allodynia (presented by PWT) and thermal hyperalgesia (presented by PWL) in MIA-induced rats were shown in (A) and (B) A:After Exosome application, PWT signifificantly increased at doses of $200 \mathrm{ug} / 100 \mathrm{uL}(\mathrm{p}$ $<0.001$ ) for at least 28 days, became signifificant within 7 days, and maintained a higher level 28 days. PWT signifificantly increased at doses of $40 \mathrm{ug} / 100 \mathrm{uL}$ or $100 \mathrm{ug} / 100 \mathrm{uL}$, became signifificant within 14 days, and maintained a higher level to 28 days. there was no difference in $100 \mathrm{ug} / 100 \mathrm{uL}$ compared with the $200 \mathrm{ug} / 100 \mathrm{uL}$ groups. $\mathrm{n}=6$ (40ug/100uL \#\# $\mathrm{P}<0.01$, \#\#\# $\mathrm{P}<0.001$; 100ug/100uL $\mathrm{P}<0.001 ; 200 u g / 100 u \mathrm{~L}, * * * \mathrm{P}<0.001$;) B:After Exosome application, PWL signifificantly increased at doses of $100 \mathrm{ug} / 100 \mathrm{uL}$ or $200 \mathrm{ug} / 100 \mathrm{uL}$ for at least 28 days, became signifificant within 7 days, and maintained a higher level 28 days. At doses of $40 \mathrm{ug} / 100 \mathrm{uL}$, became signifificant within 14 days, and maintained a higher level to 28 days. there was no difference in $100 \mathrm{ug} / 100 \mathrm{uL}$ compared with the $200 \mathrm{ug} / 100 \mathrm{uL}$ groups. $\mathrm{n}=6$ (40ug/100uL $\# \mathrm{P}<0.05$,\#\#P $\left.<0.01 ; 100 \mathrm{ug} / 100 \mathrm{uL} \$ \$ \mathrm{P}<0.01, \$ \$ \$<0.001 ; 200 \mathrm{ug} / 100 \mathrm{uL},{ }^{* *} \mathrm{P}<0.01,{ }^{* * *}<0.001 ;\right)$

Figures

Figure 2 The HE and OARSI grade was assessed in Sprague-Dawley rats.2A:Images of HE staining of knee joint specimens. Scale bar $=50 \mu m .2 \mathrm{~B}$ :After Exosome application, OARSI grade showed significant statistical difference on 28 days compared the MIA group to the Exosome groups.there was no difference between exosome groups.* represents the comparison between Exosome groups and Sham group $(* * * \mathrm{P}<0.001)$,\# represents the comparison between Exosome groups and MIA group $(\# \mathrm{P}<0.05)$

Figure 3 ATF-3 and GAP-43 protein in DRG on rats. A:After MIA application, ATF-3 expression signifificantly increased on the 14 th day after MIA injection $(P<0.001) *$ represents the comparison between MIA groups and Sham group( $* * * P<0.001)$ B:After MIA application, GAP-43 expression signifificantly increased on the 14th day after MIA injection $(P<0.001)$ * represents the comparison between MIA groups and Sham group ( $* * * \mathrm{P}<0.001)$ C:After Exosome application, ATF-3 expression signifificantly increased at doses of $100 \mathrm{ug} / 100 \mathrm{uL}$ and $200 \mathrm{ug} / 100 \mathrm{uL}$ $(\mathrm{p}<0.001)$ for at least 28 days, became signifificant within 7 days, and maintained a higher level 28 days. At doses of $40 \mathrm{ug} / 100 \mathrm{uL}$, became signifificant within 14 days, and maintained a higher level to 28 days. there was no difference in $100 \mathrm{ug} / 100 \mathrm{uL}$ compared with the $200 \mathrm{ug} / 100 \mathrm{uL}$ groups.* represents the comparison between Exosome groups and MIA group ( ${ }^{*} \mathrm{P}<0.05,{ }^{* *} \mathrm{P}$ $<0.01)$ D:After Exosome application,GAP-43 expression signifificantly increased at doses of $100 \mathrm{ug} / 100 \mathrm{uL}$ and $200 \mathrm{ug} / 100 \mathrm{uL}(\mathrm{p}<0.001$ ) for at least 28 days, became signifificant within 7 days, and maintained a higher level 28 days. At doses of $40 \mathrm{ug} / 100 \mathrm{uL}$, became signifificant within 28 days. there was no difference in $100 \mathrm{ug} / 100 \mathrm{uL}$ compared with the $200 \mathrm{ug} / 100 \mathrm{uL}$ groups .* represents the comparison between Exosome groups and MIA group $(* \mathrm{P}<0.05, * * * \mathrm{P}<0.001)$

Figure 4 ATF-3 positive neurons in DRG on MIA-induced rats. Effects of tested doses of Exosome ( $40 \mathrm{ug} / 100 \mathrm{uL}, 100 \mathrm{ug} / 100 \mathrm{uL}, 200 \mathrm{ug} / 100 \mathrm{uL}$ ) on ATF-3 positive neurons after Exosome injection were shown in (B). A:After Exosome application,ATF-3 -positive neurons on 28 days after Exosome injection in different groups. B:After Exosome application, ATF-3 positive neurons signifificantly increased at doses of $40 \mathrm{ug} / 100 \mathrm{uL}, 100 \mathrm{ug} / 100 \mathrm{uL}$ and $200 \mathrm{ug} / 100 \mathrm{uL}$ on 28 days after intra-articular Exosome. there was no difference in $100 \mathrm{ug} / 100 \mathrm{uL}$ compared with the $200 \mathrm{ug} / 100 \mathrm{uL}$ groups.* represents the comparison between Exosome groups and MIA group ( ${ }^{*} \mathrm{P}<0.05,{ }^{* * *}$ $\mathrm{P}<0.001)$ 


\section{Figures}

$1 \mathrm{~A}$

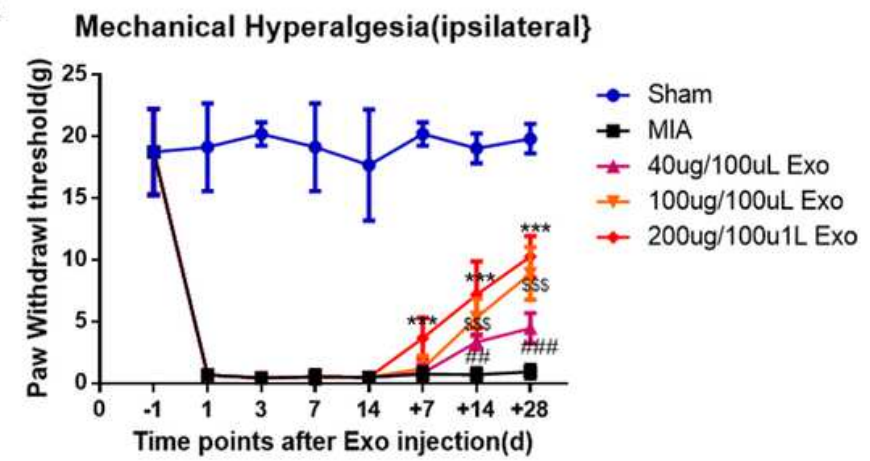

1B Thermal Hyperalgesia(ipsilateral)

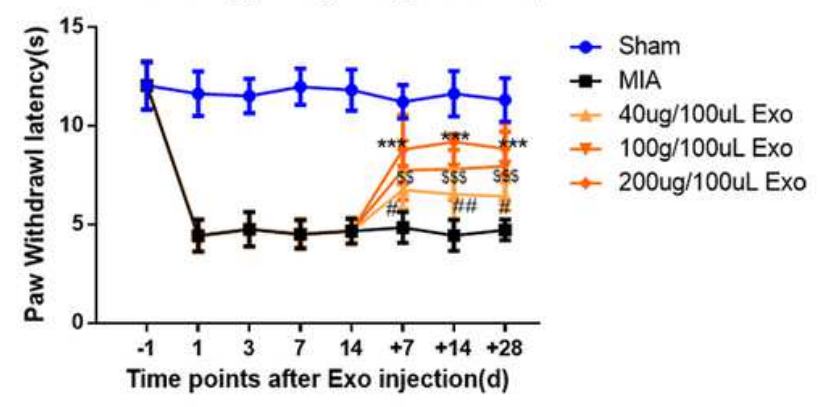

Figure 1

Pain-related behavior was assessed in Sprague-Dawley rats. Effects of tested doses of Exosome (40ug/100uL,100ug/100uL, 200ug/100uL) on mechanical allodynia (presented by PWT) and thermal hyperalgesia (presented by PWL) in MIA-induced rats were shown in (A) and (B) A:After Exosome application, PWT significantly increased at doses of $200 \mathrm{ug} / 100 \mathrm{uL}(\mathrm{p}<0.001)$ for at least 28 days, became significant within 7 days, and maintained a higher level 28 days. PWT significantly increased at doses of 40ug/100uL or $100 \mathrm{ug} / 100 \mathrm{uL}$,became significant within 14 days, and maintained a higher level to 28 days. there was no difference in 100ug/100uL compared with the 200ug/100uL groups. $n=6$ (40ug/100uL \#\#P<0.01,\#\#\#P<0.001; 100ug/100uL $P<0.001 ; 200 u g / 100 u L, ~ * * * P<0.001$;) B:After Exosome application, PWL significantly increased at doses of $100 \mathrm{ug} / 100 \mathrm{uL}$ or $200 \mathrm{ug} / 100 \mathrm{uL}$ for at least 28 days, became significant within 7 days, and maintained a higher level 28 days. At doses of 40ug/100uL,became significant within 14 days, and maintained a higher level to 28 days. there was no difference in $100 \mathrm{ug} / 100 \mathrm{uL}$ compared with the $200 \mathrm{ug} / 100 \mathrm{uL}$ groups. $\mathrm{n}=6(40 \mathrm{ug} / 100 \mathrm{uL}$ $\# P<0.05$,\#\# $<0.01 ; 100$ ug/100uL

$$
P<0.01,
$$

$\left.\$<0.001 ; 200 u g / 100 u L,{ }^{* *} \mathrm{P}<0.01, * * *<0.001 ;\right)$ 
$2 \mathrm{~A}$

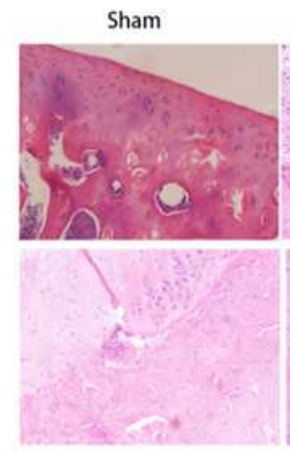

40ug/100uL Exo(28d)
MIA (7d)

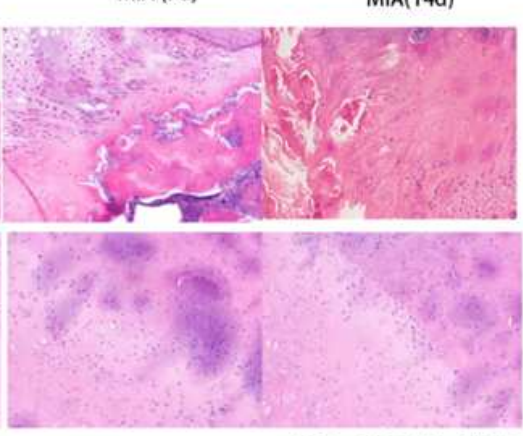

100ug/100uL Exo(28d)

200ug/100uL Exo(28d)

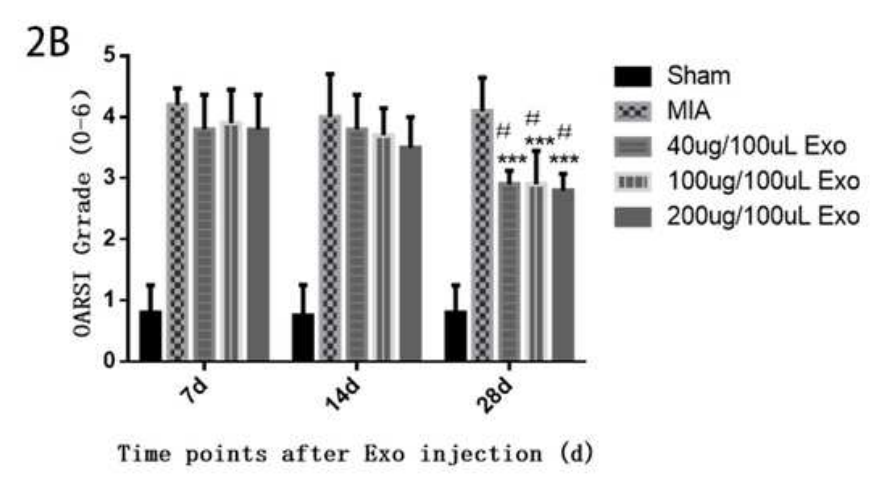

Figure 2

The HE and OARSI grade was assessed in Sprague-Dawley rats.2A:Images of HE staining of knee joint specimens. Scale bar = 50 um.2B:After Exosome application, OARSI grade showed significant statistical difference on 28 days compared the MIA group to the Exosome groups.there was no difference between exosome groups. * represents the comparison between Exosome groups and Sham group ( $* \star \star ~ P<0.001)$,\# represents the comparison between Exosome groups and MIA group $(\# \mathrm{P}<0.05)$ 

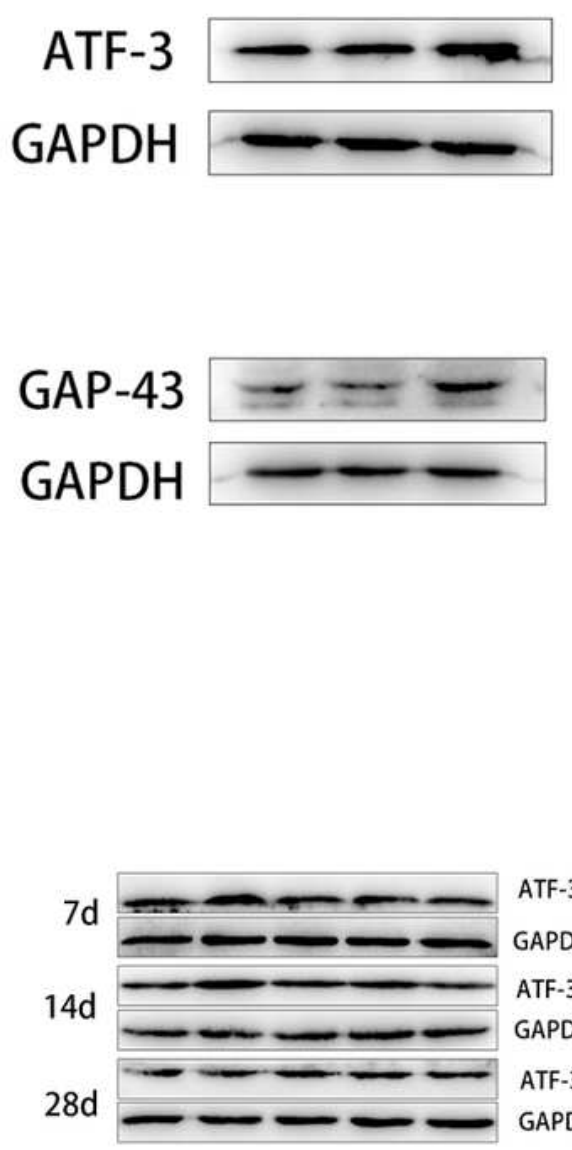
ATF-3 GAPDH ATF-3 GAPDH ATF-3 GAPDH

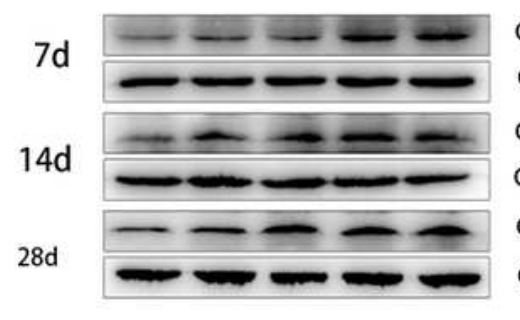
GAP-43
GAPDH
GAP-43
GAPDH
GAP-43
GAPDH

3D

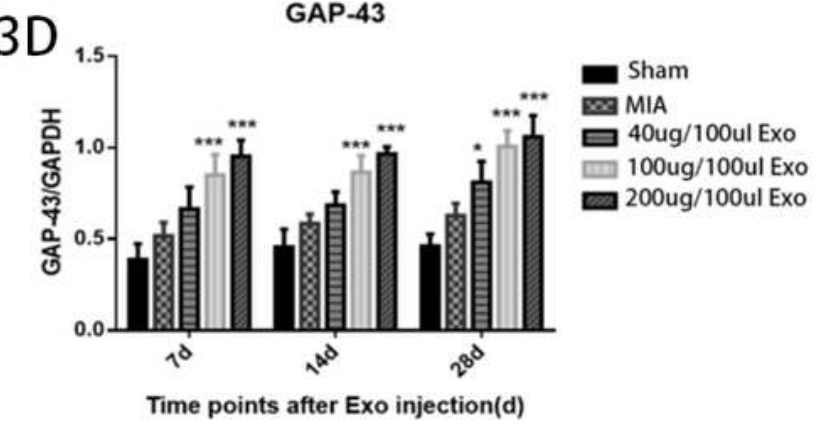

\section{Figure 3}

ATF-3 and GAP-43 protein in DRG on rats. A:After MIA application, ATF-3 expression significantly increased on the 14th day after MIA injection $(P<0.001)$ * represents the comparison between MIA groups and Sham group ( $* \star \star ~ P<0.001)$ B:After MIA application, GAP-43 expression significantly increased on the 14th day after MIA injection $(P<0.001)$ * represents the comparison between MIA groups and Sham group ( $\left.{ }^{* \star *} \mathrm{P}<0.001\right)$ C:After Exosome application, ATF-3 expression significantly increased at doses of 
100ug/100uL and 200ug/100uL ( $p<0.001$ ) for at least 28 days, became significant within 7 days, and maintained a higher level 28 days. At doses of 40ug/100uL ,became significant within 14 days, and maintained a higher level to 28 days. there was no difference in 100ug/100uL compared with the 200ug/100uL groups. ${ }^{\star}$ represents the comparison between Exosome groups and MIA group $\left({ }^{*} P<0.05\right.$, ** $\mathrm{P}<0.01)$ D:After Exosome application,GAP-43 expression significantly increased at doses of 100ug/100uL and 200ug/100uL ( $p$ <.001) for at least 28 days, became significant within 7 days, and maintained a higher level 28 days. At doses of $40 \mathrm{ug} / 100 \mathrm{uL}$, became significant within 28 days. there was no difference in $100 \mathrm{ug} / 100 \mathrm{uL}$ compared with the $200 \mathrm{ug} / 100 \mathrm{uL}$ groups .* represents the comparison between Exosome groups and MIA group $(* P<0.05$, *** $P<0.001)$
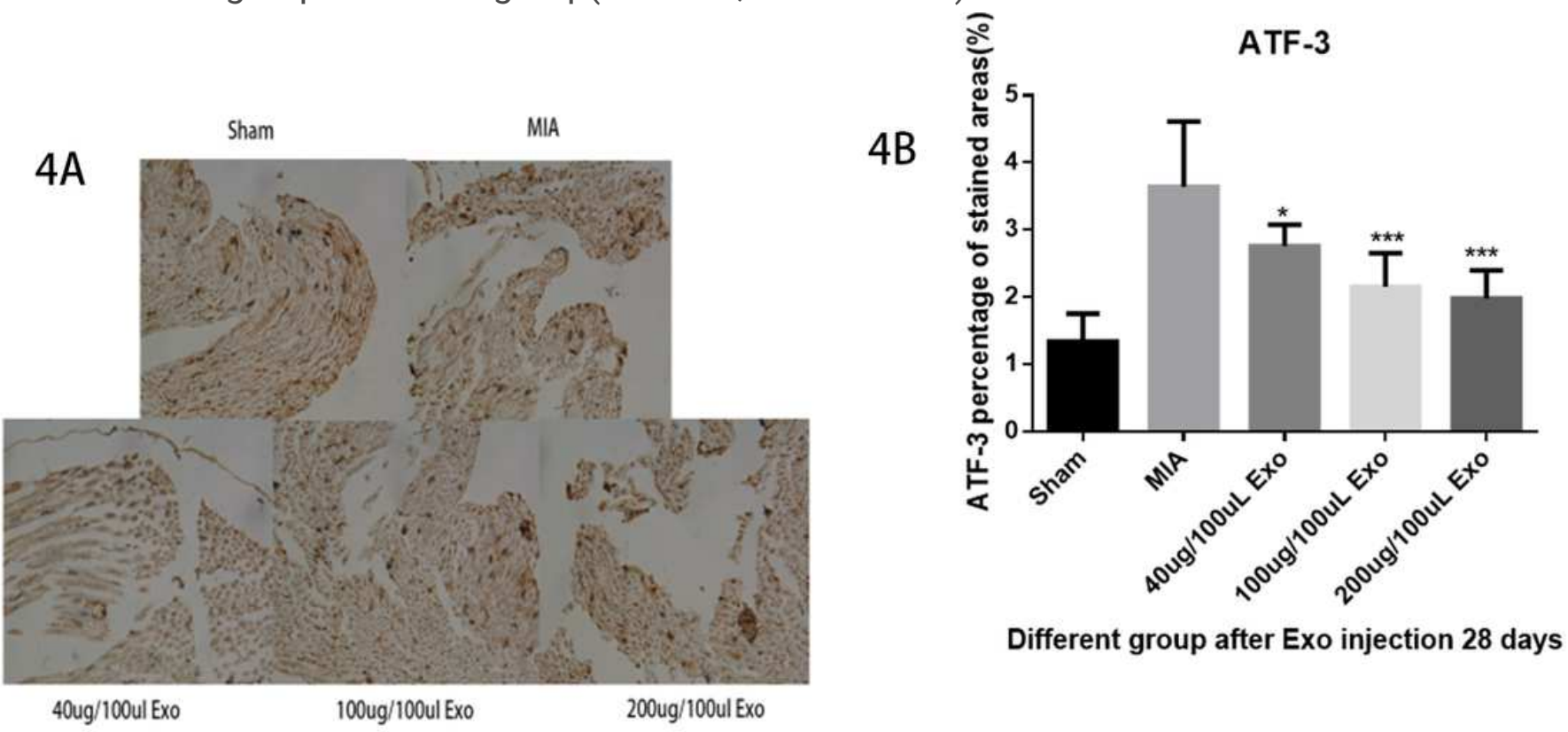

\section{Figure 4}

ATF-3 positive neurons in DRG on MIA-induced rats. Effects of tested doses of Exosome ( 40ug/100uL,100ug/100uL, 200ug/100uL) on ATF-3 positive neurons after Exosome injection were shown in (B). A:After Exosome application,ATF-3 -positive neurons on 28 days after Exosome injection in different groups. B:After Exosome application, ATF-3 positive neurons significantly increased at doses of 40ug/100uL, 100ug/100uL and 200ug/100uL on 28 days after intra-articular Exosome. there was no difference in $100 \mathrm{ug} / 100 \mathrm{uL}$ compared with the $200 \mathrm{ug} / 100 \mathrm{uL}$ groups. ${ }^{*}$ represents the comparison between Exosome groups and MIA group $(* P<0.05$, *** $P<0.001)$ 